\title{
A study of fluid flow in a MEMS designed for the determination of fluid and flow characteristics
}

\author{
N. Damean, P. P. L. Regtien \\ University of Twente, EL / MI, P.O. Box 217, 7500 AE Enschede, The Netherlands \\ fax: +31 53 4891067, e-mail: N.Damean@el.utwente.nl, P.P.L.Regtien@el.utwente.nl
}

\begin{abstract}
This paper presents a study on the hydrodynamically developed flow in hexagonal ducts, built as a MicroElectroMechanical System (MEMS) for the determination of fluid and flow characteristics. The design and operation of this structure in the framework of the new measurement concept developed previously requires detailed knowledge about the physical phenomena implied, namely about the fluid flow and heat transfer in the structure. Because the main operation of the structure is taking place in the hydrodynamically developed flow, we develop a procedure for obtaining the Poiseuille number Po versus the shape of the duct. Knowledge of this number is a main step for the design and operation of the structure. The procedure developed herein is applied also for the hexagonal ducts that are analysed in other papers and a good agreement is noticed. Main features of the procedure, are its applicability to any non-circular duct and the possibility to use a commercial software package are underlined. Finally, the next steps for the design of this structure are specified.
\end{abstract}

\section{INTRODUCTION}

Recently, the authors developed a new measurement concept $(n m c)$ [1]. Its core is an actuator-sensor structure (briefly structure). The principles regarding the design of this new structure as a MicroElectroMechanicalSystem (MEMS) are mentioned. The authors illustrated this concept with a structure designed for the evaluation of fluid and flow characteristics. New developments concerning this example are considered herein.

We consider to evaluate the fluid and flow characteristics of a single-component, laminar, Newtonian and incompressible fluid under ordinary conditions (for pressure, density, temperature, speed, typical times, etc.). Therefore, our attention is restricted to the following characteristics [2]:

- kinematic characteristics (linear velocity - shortly named velocity);

- transport characteristics (viscosity, thermal conductivity);

- thermodynamic characteristics (pressure, density, specific heat, temperature).

The outline of the paper is as follows. We give a short introduction concerning the $n m c$, underlying its role in defining and solving the measurement problem using a structure built as a MEMS. Then the a priori information we need for solving the measurement problem are defined. This information concerns the fluid flow in the structure. The description and the functionality of the structure is the subject of the next section. Since the hydrodynamically developed flow plays the most important role in this functionality, we show why the achievement of the Poiseuille number $P o$ is a crucial issue that should be managed for solving the measurement problem. The definition and the achievement of $P o$ is included in the next section. We display $P o$ versus the aspect ratio of the duct. Some conclusions, remarks and suggestions are included in the last section.

Although this paper focusses on a certain practical problem, we stress that the majority of these results might be used directly or might be adapted for various fluidic devices encountered both in micro and macro world.

\section{FORMULATION \\ OF THE MEASUREMENT PROBLEM IN THE FRAMEWORK OF THE $N M C$}

The structure is designed in the framework of the $n m c$ exposed in [1] for the simultaneous evaluation of a set of characteristics. This structure is an ensemble of electrical resistors placed together as close as possible in a duct and controlled by a computational block. The electrical resistors behave as both actuators (heaters) and sensors (temperature sensors) but they might be designed for a dedicated task as well.

The heaters are slightly modifying the temperature distribution in their vicinity depending on the characteristics mentioned above and on the heating conditions. The temperature sensors measure the temperature of certain areas around the heaters. Given the flow and thermal model of the fluid, the characteristics might be evaluated from the temperature measurements.

As mentioned above, the evaluation of the following characteristics is considered: velocity $(V)$, viscosity $(\mu)$, thermal conductivity $(k)$, density $(\rho)$ and specific heat $\left(c_{p}\right)$. The temperature characteristic $(T)$ might be evaluated using a distinct and dedicated (resistive) sensor for this task integrated in the structure. The pressure gradient along the duct $(d p / d x)$ is evaluated rather than the pressure characteristic $(p)$. For this task, 
a membrane could be used as the main component of a distinctive sensor of the structure [3]. We do not focus on these distinct and dedicated sensors (for the evaluation of a certain characteristic), considered as additional parts of the structure, because well-known solutions exist. These sensors might be used for the specific tasks in the framework of the classical measurement concept $(\mathrm{cmc})$ as described in [1].

A common point for the evaluations of the five characteristics is that they are based on the temperature measurements fulfilled by the temperature sensors as constituents of the structure. We focus only on this aspect that might be counted also like a practical application of the nmc described generally and formally in [1].

The full set of seven characteristics is not independent. The modelling of physical phenomena is a milestone in order to get proper dependencies between these characteristics [1]. These dependencies together with the temperature measurements are used to obtain the desired evaluations.

It is worth noticing that in the technical literature, similar tasks are solved through assembling together of the dedicated sensors for each characteristic. They behave as conventional stand-alone sensors in the framework of the $c m c[4-6]$.

The structure is a MEMS tailored to evaluate the desired characteristics in the framework of the $n m c$. Because of its small dimensions in comparison with the environment where the structure is used, it is assumed to evaluate these characteristics simultaneously in a single spatial point. Consequently, the characteristics are counted like spatial independent in the structure domain, that is a crucial condition for the validity of the $n m c$.

The shape of the structure is set by the silicon technology. It has a hexagonal duct etched in $<100\rangle$ silicon as a main component. The fluid flow into this particular duct has to be studied.

Ducts form the basic element of all micro scale fluidic devices like valves, mixers, pumps, fluid sensors and actuators, etc. Therefore, the present study is directly applicable to many micro scale fluidic devices [7]. The general importance of studies concerning fluid flow in non-circular ducts is obvious. Besides micro scale fluidic devices, compact heat exchangers constitute another category that benefit from the present study [8]. We are focussing only on the hydrodynamically developed flow in this work for the reasons we detail in the next section. In this respect, the Poiseuille number $P o$ (as a global characterization of the hydrodynamically developed flow) is in our attention.

Everywhere in this paper, we denote "hexagonal duct $\theta^{\circ}$ " a duct whose cross-section is a hexagon having two symmetry axes, one of the dihedral angles being $\theta^{\circ}$, as shown in Fig. 1. Frequently we refer in this paper to the hexagonal duct $60^{\circ}$ and hexagonal duct $57.47^{\circ}$.

\section{DESCRIPTION AND FUNCTIONALITY \\ OF THE ACTUATOR-SENSOR STRUCTURE}

A sketch of the structure is shown in Fig. 1. Its frame is made on a $<100>$ Si wafer covered with a Pyrex wafer, both containing an etched groove. Some electrical resistive lines are placed across the duct. For the sake of simplicity, we display only one resistive line in Fig.1. The resistive line consists of $n$ electrical resistors made of thick metal films placed along the $z$-axis over the width of the duct in the hydrodynamically developed flow region. As an example, the length of the structure along the $x$-axis is $10 \mathrm{~mm}$, its width along the $z$-axis is $1 \mathrm{~mm}$, its depth along the $y$-axis is 0.5 $\mathrm{mm}$ and $n=25$. The structure looks like a hexagonal duct defined by the orientation of the silicon crystal planes with $\theta=$ $54.74^{\circ}$.

The voltages across each resistor are measured. The computational block controls the resistors to operate as actuators or $/$ and as sensors. This block evaluates the characteristics we are looking for, using the data provided by the electrical resistors and the a priori information as: the model of the structure, the activation of the actuators, the evaluations fulfilled by the dedicated sensors and the initial information about the fluid and its flow. Therefore, the operation of the structure relies mainly on the dependencies between the temperature distribution generated by the heaters inside the duct versus the fluid and flow characteristics that are going to be evaluated.

The computational block derives the evaluations of these resistances which depend on the characteristics mentioned above. These dependencies are expressed in the stationary state as [1]:

$$
R_{i}=h_{i}\left(\left\{u_{j}, j=\overline{1, n}\right\}, \mu, k, \rho, c_{p}\right), i=\overline{1, n}
$$

where the mappings $h_{i}$ are provided by the mathematical model of the structure and the a priori information.

The model is based on the three basic equations of a fluid (namely the conservation of mass, momentum and energy, respectively), and on the equations describing the heat transfer in the fluid and in the structure. The flow we are concerned is generated by pressure gradients. This type of flow, with application primarily to ducts, is named as Poiseuille flow [9].

Consider a straight duct of arbitrary but constant shape, as illustrated in Fig. 1. After an entrance effect, more deeply in duct, the velocity becomes purely axial and varies only with the lateral coordinates; that is, $v=w=0$ and $u=u(y, z)$. The flow is then hydrodynamically developed whose basic equation is:

$$
\frac{\partial^{2} u}{\partial y^{2}}+\frac{\partial^{2} u}{\partial z^{2}}=\frac{1}{\mu} \cdot \frac{\mathrm{d} p}{\mathrm{~d} x}=\text { const }
$$

subject only to the no-slip condition on the duct surface. 


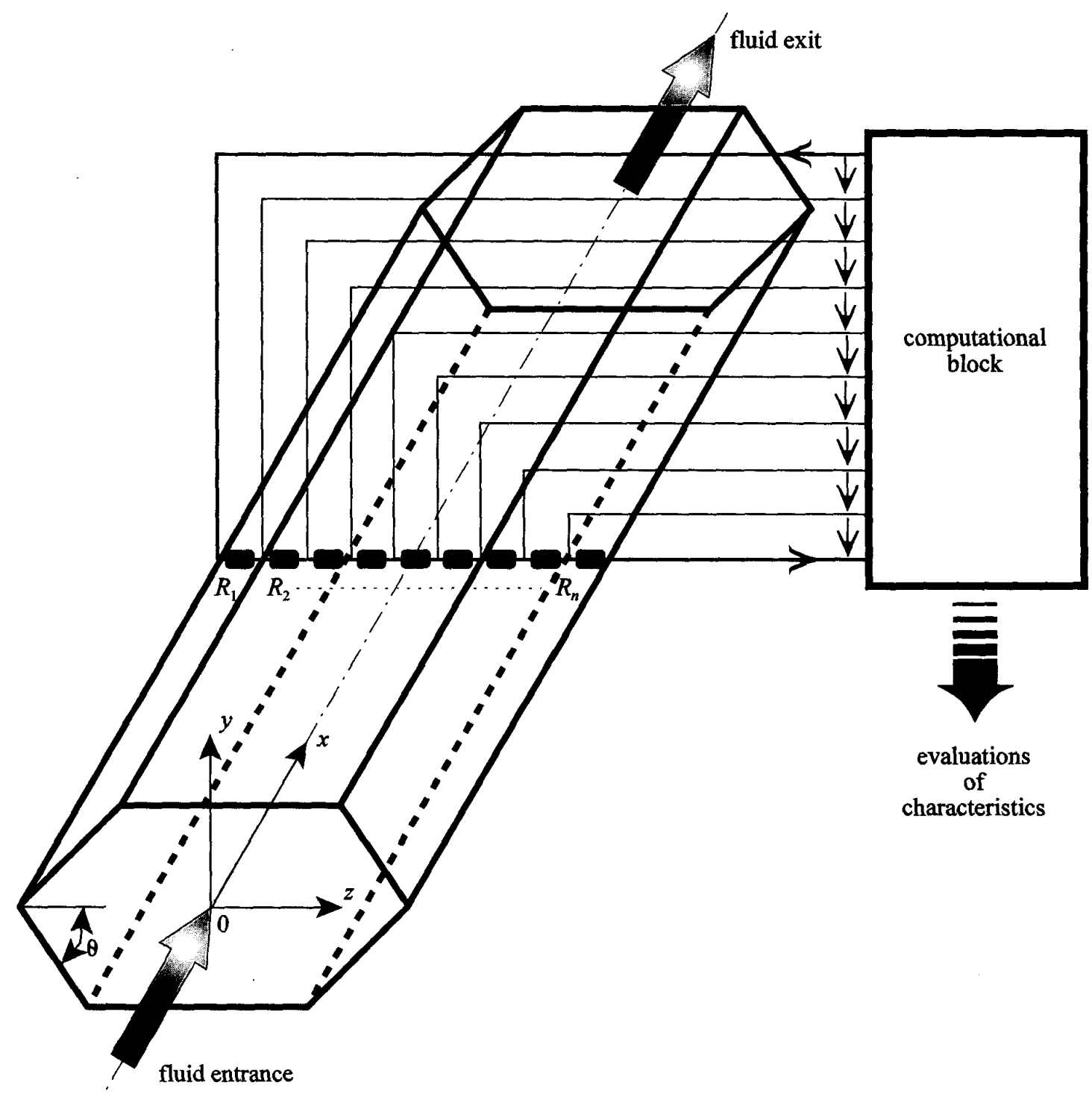

Fig. 1 Simplified sketch of the structure.

This is the classic Poisson equation and is exactly equivalent to the Poisson eq. for torsion of bars, heat transfer in plates or to membrane vibration $[2,10]$. Because of these equivalencies, the results concerning the Poisson eq. obtained in one of these areas might be immediately applied in any of these areas. Thus, many solutions for different duct shapes are known as summarized in [10 - 12]. Unfortunately, the hexagonal shape as presented in Fig. 1 is not among them, therefore the study presented herein is fully justified.

For solving the Poisson eq. for various duct shapes, a set of methods have been developed accordingly to the final utility of the solution. These are classified in exact solutions (generally obtained for some simple geometries) and approximate solutions $[10,11]$. One of the latter, achieved by the finite volume method, is used in the present work.

We use the solution of the Poisson eq. (2) for obtaining $P o$, in the view of simplifying eqs. (1) according to the measurement problem considered herein. The achievement of $P o$ for hexagonal ducts is described next. We indicate a numerical procedure used for this target. We study the effect of the mesh size and finally we display the variation of $P o$ versus the aspect ratio $\gamma$ (defined as heigh over width) of the hexagon. 


\section{POISEUILLE NUMBER}

For simplifying eqs. (1) we are proving that $\mu$ may be evaluated independently from $k, \rho, c_{p}$. We take advantage of the location of the resistive line in the hydrodynamically developed flow.

Hydrodynamically developed flow in ducts may be characterized by the Poiseuille number $P o$ [13]:

$$
P o=-\frac{1}{\mu} \cdot \frac{\mathrm{d} p}{\mathrm{~d} x} \cdot \frac{D_{h}^{2}}{2 \cdot V_{a v}}
$$

where $D_{h}$ is the hydraulic diameter and $V_{a v}$ is the cross-sectionally averaged velocity of the $u$-component of $V$

$$
V_{a v}=\frac{1}{A} \cdot \int u \cdot \mathrm{d} A
$$

and $A$ is the cross-sectional area.

$P o$ is a dimensionless constant that is independent of fluid material properties, velocity, temperature, or duct size. It is solely a mapping of the duct shape [10]. On the other hand, Po may be calculated directly for simple geometries, such as circular duct $(P o=16)$ or infinite parallel plates $(P o=24)$. For other geometries (like the geometry implied in our work), Po may be calculated numerically as shown in the next section.

Eq. (3) shows that if $P o$ is known for that duct and if the pressure gradient is evaluated by a dedicated sensor, then we get a dependence between $\mu$ and $V_{a v}$ as:

$$
\mu=h\left(V_{a v}\right)
$$

Using eq. (5), eqs. (1) are simplified as:

$$
R_{i}=H_{i}\left(\left\{u_{j}, j=\overline{1, n}\right\}, k, \rho, c_{p}\right), i=\overline{1, n}
$$

Considering the inverse of eq. (4), we get:

$$
u_{j}=g_{j}\left(V_{a v}\right), j=\overline{1, n}
$$

that shows that the samples $u_{j}$ are dependent only on the duct geometry and on the average velocity $V_{a v}$. Using eqs. (7), eqs. (6) are simplified as:

$$
R_{i}=G_{i}\left(V_{a v}, k, \rho, c_{p}\right), i=\overline{1, n}
$$

Therefore, instead of using eqs. (1) that include the $h_{i}$ mappings, we may use eqs. (5), (7) and (8), including the $h, g_{j}$ and $G_{i}$ mappings. This alternative offers an important advantage, namely it considers in a decoupled way three sets of mappings that could be derived independently. The $h$ and $g_{j}$ mappings, depending only of the geometrical characteristics of the duct and the a priori information, could be obtained independently from the measurement problem. Next, we include these mappings in the category of the a priori information. The $G_{i}$ mappings describe the dependencies between four characteristics that are going to be evaluated and $R_{i}$. These mappings are related to the measurement problem, but they are more simple than $h_{i}$.
For obtaining the $h$ mapping, $P o$ must be achieved, therefore this task is detailed next. For obtaining the $g_{j}$ mappings, the velocity profile inside the duct should be achieved. This problem will be detailed in a future paper.

It is noticed that determining $P o$ is of interest for obtaining a better understanding of the design requirements but also for testing the hydrodynamic laws for MEMS [13].

\section{A. Poiseuille number achievement}

As shown previously, Po might be derived by eq. (8) using a numerical method focussed on solving eq. (2). Because of the particular phenomenon analysed herein (fluid flow in a duct), a 3D approach seems to be inevitable, but is a time consuming approach. The inconvenience is avoided by using the corresponding Poisson eq. implied in modelling the heat transfer in a 2D plate, under steady-state conditions. That Poisson eq. is:

$$
\frac{\partial^{2} T}{\partial y^{2}}+\frac{\partial^{2} T}{\partial z^{2}}=\frac{1}{k} \cdot \dot{q}
$$

subject to the isothermal condition on the boundary of the plate. In eq. (9), $T$ has the significance of the absolute temperature, $k$ is the thermal conductivity and $\dot{q}$ is the rate of energy generation per unit volume [14].

Because the eqs. (2) and (9) are formally equivalent, the result we obtain solving eq. (9) is attributed directly to the solution of eq. (2) taking into account the conversion parameter between velocity and temperature as it is determined by the constituent terms and the boundary conditions of these eqs. The last $2 \mathrm{D}$ problem, namely eq. (9) is solved using the finite volume method by the CFD-ACE+ software package [15].

\section{B. Effect of the mesh size}

The effect of the mesh size on the Po evaluations is analysed first. The grids are distributed in a nonuniform manner with higher concentration of grids close to the boundary. A duct having a regular hexagonal cross-section is considered. Because of the symmetry, only a quarter of the hexagon is used for the numerical simulations. We have made simulations with $66 \times 66$, $88 \times 88$ and $111 \times 111$ mesh size. The results are presented in the following table in comparison with some results obtained by other authors.

Table 1. Po evaluations for regular hexagonal cross-section.

\begin{tabular}{c|ccccc}
\hline $\begin{array}{c}\text { mesh } \\
\text { size }\end{array}$ & 66 & 88 & 111 & ref. & ref. \\
& $\times$ & $\times$ & $\times$ & {$[12]$} & {$[8]$} \\
& 66 & 88 & 111 & & \\
\hline$P o$ & 15.058 & 15.059 & 15.059 & 15.054 & 14.974 \\
\hline
\end{tabular}




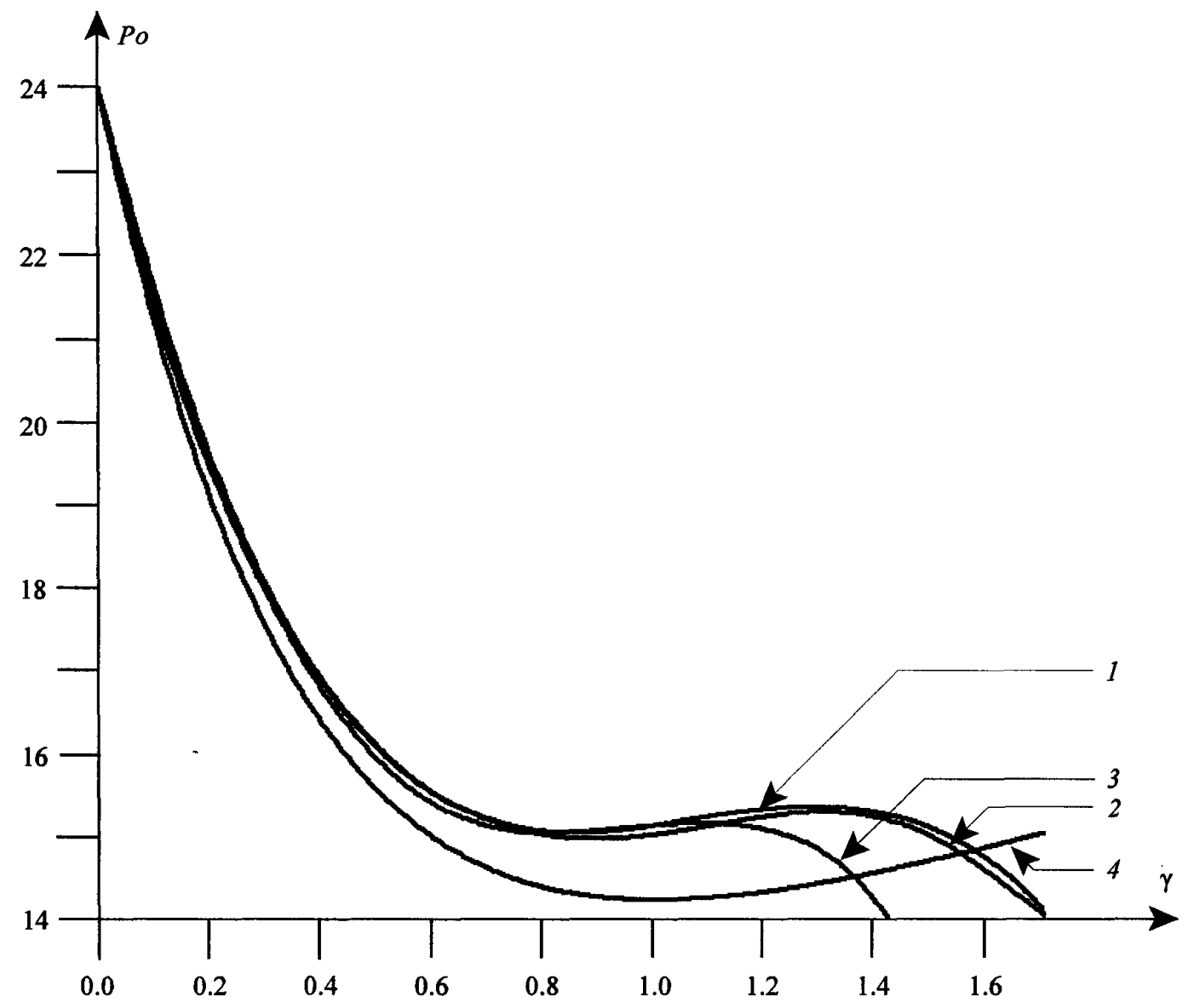

Fig. $2 P o$ versus $\gamma$ for various ducts

1: computed for the hexagonal duct $60^{\circ} ; 2$ : derived in [8] for the hexagonal duct $60^{\circ}$;

3: computed for the hexagonal duct $54.74^{\circ} ; 4$ : rectangular duct [12].

Therefore, the results we achieve are comparable with the reference results and do not depend on the mesh size. It is noticed that the reference results are obtained also numerically, but using the point-matching method [12] and the finite difference method [8].

For reducing the computation time, $66 \times 66$ mesh size grids with nonuniform distribution is used in this work. For other hexagonal ducts analysed herein, the mesh size is adjusted accordingly to the aspect ratio $\gamma$.

\section{Variation of Po versus the aspect ratio of a hexagonal duct}

For the beginning we derive numerically $P o$ for the hexagonal duct $60^{\circ}$ considering the maximum range of $\gamma$, namely $\gamma \in[0$, 1.732]. The results we obtain (curve 1) are presented in Fig. 2, in comparison with the results derived in [8] (curve 2). The target of this task is to show that the results we obtain using a commercial software package in a rather standard way are similar to the results presented in [8] where the authors have just developed a dedicated software using the finite difference method. Also we use this task for giving proof for the numerical approach we have been used in the following.

It is worth noticing that the lowest aspect ratio $\gamma=0$ is equivalent to the parallel plate where $P o=24$ [9]. The highest ratio $(\gamma=$ 1.732) corresponds to the rhombic cross-section, where the obtained value is 13.823 . It is close to 13.830 , namely the value obtained in [10] by using a discrete least squares method.

Next we derive $P o$ for the hexagonal duct $54.74^{\circ}$ and $\gamma \in[0$, 1.414]. The same procedure is used. The results are presented in Fig. 2 (curve 3). This curve is approximated by the following polynomial in $\gamma$ :

$$
\begin{aligned}
& P o=24-31.98 \cdot \gamma+67.81 \cdot \gamma^{2}-154.28 \cdot \gamma^{3}+ \\
& 260.53 \cdot \gamma^{4}-245.64 \cdot \gamma^{5}+117.06 \cdot \gamma^{6}-22.37 \cdot \gamma^{7}
\end{aligned}
$$


Eq. (10) describes the computed numerical values of $P o$ within $\pm 1 \%$ over the whole range of $\gamma$.

The values of $P o$ corresponding to $\gamma=0.5$ are 16.0644 (curve $I$ ), 16.0647 (curve 2) and 15.929 (curve 3). They are very close to 16, the value of $P o$ for the case of a circular duct. It is very probable that the theoretical value of $P o$ for the case of the hexagonal duct $60^{\circ}$ and $\gamma=0.5$ is 16 , but this hypothesis should be confirmed or infirmed by further theoretical study. On the other hand, the value of $P o$ we obtain for the hexagonal duct $54.74^{\circ}$ and $\gamma=0.5$ is about 16 . Therefore, from a practical point of view, we might approximate the hexagonal duct $54.74^{\circ}$ at $\gamma=$ 0.5 by a circular one, when we make global estimations using eq. (3). This remark underlines the validity of the results obtained by some authors who used this approximation even without proof $[16,17]$.

Also, it is noticed that some authors are approximating the hexagonal duct $54.74^{\circ}$ with a rectangular one [18]. The $P o$ values for the rectangular duct (curve 4) are smaller than the values for the hexagonal duct $54.74^{\circ}$, therefore this approximation should be avoided.

\section{CONCLUSIONS}

The hydrodynamically developed flow in hexagonal ducts is analysed in this paper. Special attention is dedicated to the hexagonal duct $54.74^{\circ}$. This type of hexagonal duct is encountered in many micro scale fluidic devices. In this respect, we have developed a procedure for obtaining $P o$ in hexagonal ducts. The results we have obtained using this procedure are compared with similar results obtained for other shapes and the validity of the present procedure is proven.

The procedure we have developed and used for hexagonal ducts might be used for other kind of non-circular ducts. Moreover, even if the immediate applications of these results are for the micro scale fluidic devices, their general applications are much extensive, namely for the non-circular ducts used also in the macro world.

\section{ACKNOWLEDGMENTS}

This work is funded by the Dutch Technology Foundation (STW) under the project TEL66-4408: Resistive sensor-actuator structures for the determination of fluid and flow parameters.

\section{REFERENCES}

[1] N. Damean, P. P. L. Regtien, "Measurement concepts - From classical transducers to new MEMS", Measurement $J$., in press.

[2] F. M. White, Viscous Fluid Flow, 2nd ed., McGraw-Hill, New York, 1991.

[3] P. J. French, P. M. Sarro, "Surface versus bulk micromachining: the contest for suitable applications", J. Micromech. Microeng., 8, pp. 45-53, 1998.

[4] P. Norlin, O. Öhman, B. Ekström, L. Forssén, "A chemical micro analysis system for the measurement of pressure, flow rate, temperature, conductivity, UV-absorbtion and fluorescence", Sensors and Actuators, B, 49, pp. 34-39, 1998.

[5] E. Yoon, K. D. Wise, "An integrated mass flow sensor with onchip CMOS interface circuitry", IEEE Trans. on Electron Devices, vol. 39, no. 6, pp. 1376-1386, 1992.

[6] V. Spiering, P. Bergveld, M. Elwenspoek, A. van der Berg, "A $\mu$ sensor array in a fluidic system for space applications", in Proc. of the 1996 National sensor conference, Delft, pp. 143-146, 1996.

[7] M. Elwenspoek, T. S. J. Lammerink, R. Miyake, J. H. J. Fluitman, "Towards integrated microliquid handling systems", J. Micromech. Microeng., 4, pp. 227-245, 1994.

[8] R. Sadasivam, R. M. Manglik, M. A. Jog, "Fully developed forced convection through trapezoidal and hexagonal ducts", Int. J. Heat Mass. Transfer, vol. 42, pp. 4321-4332, 1999.

[9] F. M. White, Fluid Mechanics, 4th ed., WCB McGraw-Hill, Boston, 1999.

[10] R. K. Shah, A. L. London, Laminar Flow Forced Convection in Ducts, Academic Press, New York, 1978.

[11] R. K. Shah, "Laminar flow friction and forced convection heat transfer in ducts of arbitrary geometry", Int. J. Heat Mass Transfer, vol. 18, pp. 849-862, 1975.

[12] W. M. Rohsenow, J. P. Harnett, Y. I. Cho, Handbook of Heat Transfer, 3rd ed., McGraw-Hill, 1998.

[13] J. N. Zemel, R. Furlan, "Microfluidics", in Handbook of Chemical and Biological Sensors, R. F. Taylor, J. S. Schultz, Eds. IOP Publishing, 1996.

[14] F. P. Incropera, D. P. DeWitt, Fundamentals of Heat and Mass Transfer, 4th ed., John Wiley \& Sons, New York, 1996.

[15] CFD Research Corporation, CFD-ACE + software package, 215 Wynn Drive Huntsville, AL 35805, USA

[16] M. Elwenspoek, T. S. J. Lammerink, "Theory of thermal flow sensors", Proc. of the 13th Europ. conf. on solid-state transducers, Eurosensors XIII, pp. 317-318, 1999.

[17] T. S. J. Lammerink, N. R. Tas, M. Elwenspoek, J. H. J. Fluitman, "Micro-liquid flow sensor", Sensors and Actuators, A, 37-38, pp. 45-50, 1993.

[18] J. Branebjerg, O. S. Jensen, N. G. Laursen, O. Leistiko, H. Søeberg, "A micromachined flow sensor for measuring small liquid flows", Proc. of the 6th int. conf. on solid-state sensors and actuators, Transducers '91, pp. 41-44, 1991. 